\title{
SUSCEPTIBILIDAD A LA EROSIÓN HIDRICA DE SUELOS EN LA ZONA ANDINA DEL DEPARTAMENTO DEL CAUCA COLOMBIA
}

\author{
SUSCEPTIBILITY TO WATER EROSION OF SOILS IN THE ANDEAN REGION \\ OF CAUCA DEPARTMENT, COLOMBIA
}

Fernando, A. Muñoz. G' ${ }^{1}$ Edier, H. Pérez², Juan, D. Otero, $\mathrm{S}^{1}$

${ }^{1 .}$ Estudiante Doctorado Ciencias Ambientales, Universidad del Cauca. E-mail: famunozg@gmail.com

2. Grupo de Estudios Ambientales, Universidad del Cauca.

Recibido: 20 de Septiembre de 2014

Aceptado: 15 de Octubre de 2014

*Correspondencia del autor:amunozg@gmail.com

\section{RESUMEN}

En este trabajo se evaluó la susceptibilidad de suelos a la erosión hídrica en la parte media de la cuenca río Las Piedras, donde predominan áreas de pastura y cultivos. Los instrumentos básicos para la investigación fueron mini-simuladores de lluvia que funcionan bajo el sistema de goteo, apoyado con análisis de propiedades físicas y químicas de suelos y bases cartográficas. Las muestras de suelo y escorrentía superficial fueron analizadas en los laboratorios de la Universidad del Cauca, siguiendo los procedimientos de análisis de suelos y agua del IGAC y Standard-methods. Los análisis reportaron para estos suelos una textura franco-arenosa, humedad de campo entre $39-51 \%$; el pH de 4.8 a 5.5 correspondiente a suelos ácidos, con porcentaje de CO superior al 5\% y CIC entre 36$46 \mathrm{meq} / 100 \mathrm{cc}$. La cantidad de suelo erodado osciló entre 0.43 a $7.8 \mathrm{~g}$ para cultivo y $0,3 .-5,78 \mathrm{~g}$ en pastura a una intensidad de $100 \mathrm{~mm} \mathrm{~h}^{-1}$; evidenciando la influencia de las precipitaciones sobre la susceptibilidad a la erosión hídrica y su relación con las características físicas y químicas del suelo en los diferentes usos.

Palabras claves: Andino, Usos del suelo, Escorrentía, Suelo erodado.

\begin{abstract}
In this study, an evaluation of the susceptibility to water erosion of soils in the central section of the Las Piedras river basin, where areas devoted to pasture and crops predominate was made. The basic tools for the research were mini rainfall simulators, operating under the drip system, supported by the analysis of soil physical and chemical properties and map databases. The soil samples and surface runoff were analyzed in the laboratory at University of Cauca following the soil and water analysis procedures of IGAC and Standard Methods. Analysis of these soils reported a sandy-loam texture, field humidity between $39-51 \%$; $\mathrm{pH}$ from 4.8 to 5.5 , corresponding to acid soils, with a $\mathrm{CO}$ percentage above $5 \%$ and $\mathrm{CEC}$ between $36-46 \mathrm{meq} / 100 \mathrm{cc}$. The quantity of eroded soil ranged from 0.43 to $7.8 \mathrm{~g}$ for crops and 0.3 to $5.78 \mathrm{~g}$ in pasture at an intensity of $100 \mathrm{~mm} \mathrm{~h}-1$, showing the influence of rainfall in the susceptibility to water erosion physical and chemical characteristics of the soil and the different land uses.
\end{abstract}

Keywords: Andean, Land use, Runoff, Eroded soil. 


\section{INTRODUCCION}

La intensificación del uso y manejo inapropiado del suelo han generado cambios físicos, químicos y biológicos en los ecosistemas altoandinos de Colombia debido a las fuertes roturaciones, el pastoreo de ganado, la mecanización y el uso de agroquímicos en las zonas agrícolas, favorecen e incrementan su vulnerabilidad frente a la erosión hídrica en estos ecosistemas, donde el incremento de la escorrentía superficial que arrastra suelo, materia orgánica, nutrientes y agroquímicos afectan el recurso hídrico superficial, la capacidad productiva del ecosistema y la salud de los recursos. Según (IDEAM, 2001) en Colombia más de 2'300.000 ha son erosionadas, a una escala superior a 1.8 t. $\mathrm{km}^{2}$ año(1).

En el Departamento del Cauca los procesos de erosión actúan con más intensidad especialmente en la zona altoandina debido a las fuertes pendientes que presentan sus zonas montañosas, al material parental de los suelos a las características climáticas (lluvias de alta intensidad), al uso y manejo inadecuado de los mismos $(2,3)$ que impactan directamente los agroecosistemas por un transporte continuo de suelo y nutrientes generando alteraciones en la actividad productiva, la población y los recursos naturales. La degradación ambiental es uno de los problemas más comunes en áreas rurales causadas principalmente por actividades humanas ligadas a sistemas de producción extensiva e intensiva, bien sea de tipo agrícola, ganadero o minero (4).

Otras investigaciones corroboran a partir de modelamiento del clima regional que, efectivamente, con cambios locales en el uso del suelo ocurren cambios de las variables climatológicas en la escala local (5).

Mediante el desarrollo de esta investigación fue posible realizar un acercamiento al conocimiento de cómo se dan la relaciones e interacciones naturales y antrópicas en los agroecosistemas altoandinos, en torno a un recurso base para la vida como el suelo en la subcuenca del río Piedras, la información obtenida tiene como fin sentar las bases para los tomadores de decisiones a nivel local, regional y nacional e impactar en el buen manejo de este recurso buscando la sostenibilidad para los agroecosistemas, beneficiando las comunidades presentes en la zona. Identificando factores que estén causando impacto en los suelos de estos y más aún cuando se convierten en sistemas productivos. Por lo anterior mencionado es necesario demostrar el deterioro real para crear conciencia, cambio y soluciones en y desde las comunidades.

Finalmente, es importante recalcar que todos los esfuerzos que vayan encaminados en caracterizar, entender, complementar y aportar a las diferentes problemáticas presentes en zonas con suelos altoandinos son fundamentales, para la orientación de sus habitantes en términos de procesos de gestión de los recursos, donde se incluya su aprovechamiento y al mismo tiempo su conservación para generaciones futuras, favoreciendo el auto sostenimiento, seguridad y soberanía alimentaria.

\section{MATERIALES Y METODOS}

La zona de estudio se encuentra ubicada sobre la cuenca del río Piedras en los municipios de Popayán y Totoró, Departamento del Cauca, Colombia. Sobre el flanco occidental de la cordillera Central, entre las coordenadas $76^{\circ} 31^{\prime} 10^{\prime \prime}$ al Oeste de Greenwich y $2^{\circ} 21^{\prime}$ $45^{\prime}$ "de latitud Norte (Fig. 1). Su extensión es de 6.626 hectáreas, con relieves ligeramente ondulado, ondulado, quebrado y escarpado, los suelos que se encuentran en el área conforman los: Aquandic Cryaquents, Typic Cryaquents, Andic Haplumbrepts, cuyas características de profundidad van desde moderadamente profundos a superficiales y muy superficiales, bien a excesivamente drenados, suelos muy evolucionados, fuertemente ácidos, con altos contenidos de aluminio intercambiable, alta capacidad de intercambio catiónico, de fertilidad baja, con procesos acelerados de pérdidas por erosión laminar. Actualmente se encuentran cubiertos con pastos naturales para ganadería extensiva con baja capacidad de carga; al igual que rastrojos de porte mediano (6).

Las actividades desarrolladas se basaron en la utilización de simuladores de lluvia en dos usos de suelo cultivo y pastura, para determinar escorrentía superficial, suelo erodado y perdida de nutrientes; apoyados en las características físicas y químicas de los suelos aplicando las metodologías reportadas en (7).

El análisis de suelos y susceptibilidad a la erosión hídrica, están determinados por la variabilidad de las lluvias naturales en el Departamento del Cauca, así como los costos y la dificultad de crear instalaciones 


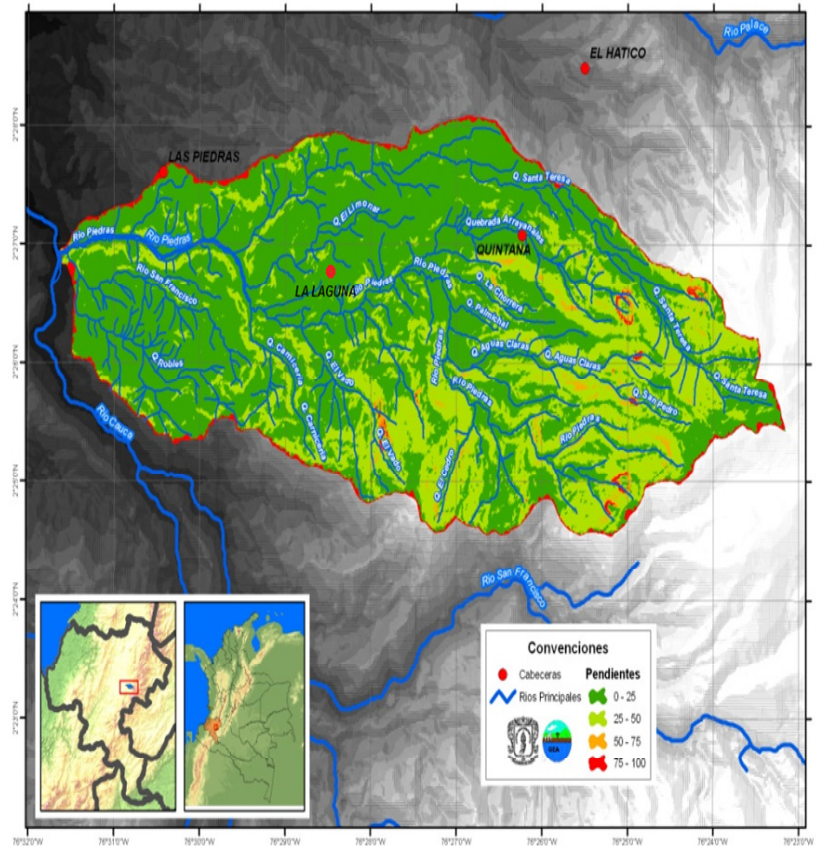

Figura. 1. Localización de área de estudio, subcuenca río Las Piedras. Fuente: Grupo de Estudios Ambientales, Universidad del Cauca

en campo de forma permanente. De esta forma la metodología planteada en la subcuenca del río Las Piedras, se basó en los estudios realizados por $(8,12)$ para susceptibilidad de los suelos a la erosión y transporte de nutrientes o agroquímicos tanto en la escorrentía superficial como en el suelo arrastrado para ecosistemas de alta montaña mediante la utilización de simuladores de lluvia que funcionan bajo el sistema de goteo,a condiciones controladas (13). El análisis de los diferentes factores de forma individual por usos de suelo permite generar datos y resultados rápidos, eficientes y reproducibles a bajo costo, considerando la pendiente, el tipo de vegetación (edad de la explotación, cultivos anteriores), el manejo previo (fertilización, preparación del suelo), las características físicas y químicas de los suelos, la presencia de rocas y cuerpos de agua, entre otros factores básicos necesarios para evaluar la erosión hídrica.

En las pruebas experimentales de campo se utilizó el minisimulador de lluvia en sistemas con cultivo y pastura, donde se evalúa el efecto de la lluvia a intensidades de $100 \mathrm{~mm} / \mathrm{h}$, durante media hora de acuerdo a lo propuesto por Morgan (1986) donde plantea que la mayor erosión se presenta en las lluvias naturales con láminas entre 30 y $60 \mathrm{~mm}(14)$.
Se estableció un diseño experimental teniendo en cuenta los dos tipos de pendiente $(>25 \mathrm{y}<50 \%)$, el uso y manejo del suelo (cultivo y pastizal), la intensidad de la lluvia $\left(100 \mathrm{~mm} \mathrm{~h}^{-1}\right)$ y un diámetro de gota de lluvia de $(2.75 \mathrm{~mm})$ más el tiempo requerido para cada simulación, media hora (30 minutos) con toma de muestra cada cinco minutos 3 repeticiones para cada punto de muestreo durante 6 meses para un total de 72 muestreos al azar en cuatro parcelas de 5 x 10 metros.

De otro lado las muestras de escorrentía superficial obtenidas se analizaron por medio del potenciómetro METTER TOLEDO MA235 pH/Ion Analyzer, Conductividad por medio de la sonda multíparametrica tipo YSI incorporated y la Determinación de Nitrógeno y Fosforo se realizóo mediante la técnica del test selectivo en cubetas para fosforo y nitrógeno respectivamente, en Spectroquant NOVA 60, con el método colorimétrico (APHA, AWWA, WPCF). La textura se determinó bajo el método hidrométrico o de Bouyoucos, la densidad por el método del cilindro, la humedad de campo se determinó tomando una muestra de suelo húmedo $10 \mathrm{~g}$, posteriormente se mantuvo a $105^{\circ} \mathrm{C}$ durante 24 horas. Porosidad mediante la diferencia del valor de la densidad real y la densidad aparente, el pH se determina con un potenciómetro, la determinación del nitrógeno y fosforo total presente en el suelo se realizó por el método kjeldhal, la determinación de aluminio $\left(\mathrm{Al}^{+3}\right)$ intercambiable se realizó desplazando los iones presentes en el suelo para luego titular (7).

\section{RESULTADOS Y DISCUSION}

Análisis de propiedades físicas y químicas del suelo En los resultados para determinar textura se observa altos porcentajes de arena para los dos suelos. El contenido de limos es mayor en pastura, también se detectan bajos niveles de arcillas en los dos agroecosistemas. Estos resultados indican el posible grado de erosión sobre todo en los suelos dedicados a cultivo durante mucho tiempo y esta acuerdo a lo planteado en sistema de clasificación textural de suelos del U.S. Department of Agriculture (USDA)(15). 
Tabla1. Caracterización Propiedades físicas de los suelos

\begin{tabular}{ccc}
\hline Variable (\%) & Pastura & Cultivo \\
\hline Porosidad & 75,6 & 65,2 \\
Humedad & 30,2 & 20,8 \\
Arena & 76,1 & 78,1 \\
Limo & 16,6 & 16,1 \\
Arcilla & 7,3 & 5,8 \\
Textura & F. A & F. A \\
\hline
\end{tabular}

F.A: Textura franco Arenosa

La porosidad en los dos agroecosistemas evaluados es alta según Montenegro (16) al ser superior al 60\%, resultado esperado por la estrecha relación con la textura franco-arenosa. En pastura se presenta mayor porosidad inicialmente por la cobertura vegetal, ya que las raíces forman poros grandes y abiertos, haciendo que el agua y el aire circulen con mayor facilidad, permitiendo una mayor infiltración, para pastura también se encontraron ciertos organismos edáficos que perforan la tierra formando poros.

Los porcentajes más altos de humedad se dieron en pastura, debido a la menor presencia de arena, alto contenido de materia orgánica (MO) y porosidad reteniendo mayores cantidades de agua. Además la cobertura vegetal controla la evaporación restringiendo el paso de la humedad al exterior; en contraste el cultivo con suelo desnudo hace una evaporación más rápida por la exposición directa a factores medioambientales como el aire, el sol entre otros.

La MO como se observa en la tabla 2, a profundidad de $0-5 \mathrm{~cm}$ presenta valores altos en los dos agroecosistemas, clasificados como suelos orgánicos al presentar más del 10\%, influyendo en las propiedades físicas, químicas y en la actividad biológica del suelo, por lo cual afecta la fertilidad del mismo. En cuanto a la relación $\mathrm{C} / \mathrm{N}$ se encuentra mejor en suelos de cultivados que en suelos de pastura, dada la adición de abonos.

En cuanto a la acidez (Tabla 2), se ubica según la clasificación U.S.DA. en (17) como suelos ácidos al presentar un $\mathrm{pH}$ entre 4,5 y 5,5. Además de la acidez generada por su origen volcánico, la adición inadecuada de abonos e insumos químicos para el cultivo aportan acidez al suelo (8); en el cultivo se esperaba un $\mathrm{pH}$ más neutro debido a la utilización de cal y agroquímicos antes de sembrar, pero las características texturales dan un indicio de cómo los agroquímicos se infiltran o son arrastrados por escorrentía superficial.

El intercambio catiónico presenta valores altos para las dos zonas al estar dentro del rango de 30 a 50 $\mathrm{meq} / 100 \mathrm{~g}$ debido a los altos contenidos de MO, respecto a su relación del CIC con el $\mathrm{pH}$ menor de 5.5 no es muy clara ya que el catión dominante en estos suelos es el aluminio siendo tóxico para las plantas y el suelo asociándose con deficiencias de $\mathrm{P}, \mathrm{Ca}$ y $\mathrm{Mg}$.

Los altos niveles de nitrógeno en los dos agroecosistemas son atribuibles a la gran adición de fertilizantes nitrogenados y al alto contenido de MO. $(18,19)$. En cuanto a los valores de fósforo disponible (Tabla 2) son bajos al estar por debajo de $30 \mathrm{mgL}^{-1}$ (19), atribuido a la fuerte acidez de estos suelos derivados de cenizas volcánicas; de acuerdo a (20) el fósforo se enlaza con la superficie reactiva de las alófanas y los complejos de humus-aluminio, afectando el desarrollo y el crecimiento de la plantas.

Tabla 2. Caracterización de las propiedades químicas del suelo.

\begin{tabular}{ccc}
\hline Variable & Pastura & Cultivo \\
\hline MO (\%) & 11,57 & 12,5 \\
$\mathrm{CO}(\%)$ & 6.71 & 7.25 \\
Fosforo (\%) & 3,95 & 7,22 \\
$\mathrm{~N}(\%)$ & 1,8 & 1,2 \\
$\mathrm{CIC}(\mathrm{meq} / 100 \mathrm{~g})$ & 39,12 & 43,5 \\
$\mathrm{Al}(\mathrm{meq} / 100 \mathrm{~g})$ & 1,22 & 0,17 \\
$\mathrm{pH}$ & 5,5 & 5,1 \\
$\mathrm{C} / \mathrm{N}$ & 3,72 & 6,04 \\
\hline
\end{tabular}

Comportamiento de suelo erodado y escorrentía superficial

Durante las pruebas de simulación de lluvia se evidencio que en los suelos de cultivo la escorrentía superficial y el suelo erodado se incrementan (Figura 2 ). En el mes de noviembre se registraron los mayores niveles de suelo erodado $8.0 \mathrm{~g}$ y de escorrentía superficial $2000 \mathrm{~mL}$, cantidades estas que se deben a las altas precipitaciones naturales en este mes (300 a $500 \mathrm{~mm}$ ) donde la humedad del suelo supero el $83 \%$ que favoreció la escorrentía superficial. 


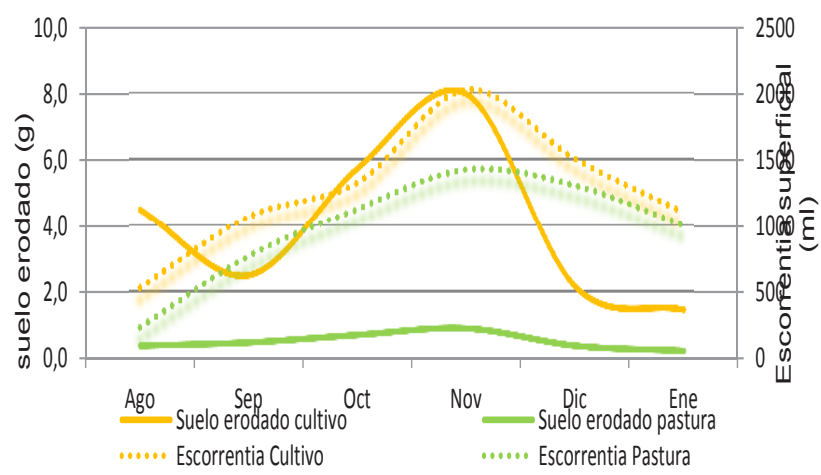

Figura 2. Suelo erodado y escorrentía

En el sistema con cubertura vegetal de pastos para el mes de agosto se presentaron altos niveles de escorrentía( $1490 \mathrm{~mL})$ y muy bajos de suelo erodado $(0.78 \mathrm{~g})$ mostrando una clara diferencia entre los dos sistemas y la importancia de la cobertura vegetal; la humedad bajó levemente $(83.18 \%)$.

\section{Propiedades físicas y químicas de la escorrentía superficial.}

La conductividad en la solución del suelo se registró entre 0.0015 y $0.028 \mathrm{mS} / \mathrm{cm}$, valores que están muy relacionados con la preparación del terreno para el cultivo, la adición de fertilizantes como 10-30-10 (N-P-K) y la variabilidad de los niveles de precipitación favoreciendo la escorrentía, al igual que el $\mathrm{pH}$ neutro entre (6.1-6.7 Cultivo y 6.4-6.8 Pastura). Es importante notar que la concentración de $\mathrm{P}-\mathrm{PO}^{3-}$ en escorrentía superficial, se mostró muy bajo (0.09-0.18 Cultivo y 0.08-0.09 mg/L Pastura) en el transcurso de la investigación se observa que los valores no alcanzaron $0.1 \mathrm{mgL}^{-1}$ lo cual es muy bajo y no se presenta arrastre de fosfatos por escorrentía superficial. El cultivo reportó mayor cantidad de arrastre de nitratos con un promedio de $1,10 \mathrm{mg} / \mathrm{L}$ con respecto a los $0,90 \mathrm{mg} / \mathrm{L}$ que se encontraron para pastura. El arrastre de este nutriente fue significativo, posiblemente por la extracción por parte del cultivo y el lavado por erosión, ya que los nitratos presentan alta solubilidad en este tipo de suelos.

\section{Relación de usos-erosión}

El factor más influyente para los usos fue el manejo del suelo que se presenta al dejar el suelo descubierto durante la preparación del terreno para el inicio del cultivo donde se favorece la disgregación y arrastre por escorrentía superficial. En algunos casos las pendientes pronunciadas tienen longitudes mayores de $15 \mathrm{~m}$ favoreciendo la velocidad de la escorrentía superficial, pasando de un factor de trasporte a uno de desprendimiento provocando una erosión más severa.

\section{CONCLUSIONES}

El suelo en preparación para cultivo presentó los valores más altos de suelo erodado y escorrentía superficial, en comparación con la pastura, también se comprobó la relación directamente proporcional entre la escorrentía superficial y el suelo erodado, de igual forma en este agroecosistema los nitratos tuvieron un fuerte decrecimiento por la acidez de los suelos aumentando la solubilidad de este nutriente y facilitando su arrastre por escorrentía superficial. La acidez mostrada se da inicialmente por ser suelos de origen volcánico, sin embargo los efectos del manejo, la adición de fertilizantes, erosión, niveles medios de aluminio y materia orgánica generaron una notable tendencia ácida.

Es evidente como prácticas inadecuadas de cultivo están generando degradación por escorrentía superficial en suelos altoandinos, repercutiendo directamente en la seguridad alimentaria y la oferta ambiental de estos ecosistemas.

\section{AGRADECIMIENTOS}

Agradecemos especialmente al Departamento Administrativo de Ciencia, Tecnología e Innovación (COLCIENCIAS), al Ministerio de Agricultura y Desarrollo Rural, a la Universidad del Cauca y a la Vicerrectoría de Investigaciones por su financiación, y en particular al Grupo de Estudios Ambientales. 


\section{BIBLIOGRAFÍA}

1. IDEAM. La erosión del suelo IDEAM. 2001.

2. IGAC. Estudio General de Suelos y Zonificacion de Tierras del Departamento del Cauca. (IGAC) IGAC, editor. Bogotá2009.

3. Muñoz F. Evaluación de la susceptibilidad a la erosión en dos tipos de agroecosistemas alto andinos en la cuenca del río Palacé. Popayá: Universidad del Cauca; 2007.

4. Tan Z, Lai, R. . Carbon sequestration potential estimates with changes in land use and tillage practice in Ohio. Agriculture, Ecosystems and Environment 2005:111, 40-52.

5. Rodríguez E, N., Pabón-Caicedo J.D., Bernal-Suárez N.R. y Martínez-Collantes J. Cambio climático y su relación con el uso del suelo en los Andes colombianos. Instituto de Investigación de Recursos Biológicos Alexander von Humboldt, editor. Bogotá, D. C., Colombia. 2010.

6. CRC. Plan de ordenación y manejo de la subcuenca hidrográfica del rio Las Piedras. In: Cauca, editor.: Corporacion Autonoma Regional de Cauca (CRC); 2006. p. 451.

7. IGAC. Manual de metodos analiticos de laboratorio de suelos. Codazzi IGA, editor. BogotaColombia2007.

8. Otero JD. Evaluación del arrastre de nutrientes en zona de ladera generado por escorrentía superficial en dos agroecosistemas dentro de la zona de amortiguación del Parque Nacional Natural Puracé. [Trabajo de Grado]. Popayán: Universidad Del Cauca; 2009.

9. Otero JD, Figueroa A, Muñoz FA, Peña MR. Loss of soil and nutrients by surface runoff in two agro-ecosystems within an Andean paramo area. Ecological Engineering. 2011;37(12):203543.

10. Muñoz FA. Evaluación de la susceptibilidad a la erosión en dos tipos de agroecosistemas alto andinos en la cuenca del río Palacé [Trabajo de Grado]. Popayán: Universidad Del Cauca. Facultad de Ciencias Naturales Exactas y de la Educación. Departamento de Química; 2007.

11. Cobo Q. Diseño, construcción y evaluación de un mini simulador portátil de lluvia para estudios de susceptibilidad a erosión en laderas [Trabajo de Grado]. Santiago de Cali - Palmira: Universidad del Valle - Universidad Nacional; 1998.

12. Torres E. Evaluación de la susceptibilidad a la erosión y de las características asociadas (infiltración, y escorrentía) de los suelos de ladera en el departamento del Cauca, mediante un mini simulador de lluvia [Trabajo de Grado]. Palmira: Universidad del Valle, Universidad Nacional de Colombia; 2000.

13. Pérez EH, Paez MIM, Figueroa CA, . Effect of humidity and temperatura on dissipation of chlorpyrifos and diazinon in andic soils, Cauca, Colombia. Asian Journal of Chemistry. 2013:9208-12.

14. Morgan RPC. Soil Erosion and Conservation. New York: Longman. 1986.

15. USDA. Keys to soil taxonomy. . United States Department of Agriculture. 2006;10 Th Ed:332.

16. Montenegro GH. Interpretación de las propiedades físicas del suelo (Textura, Estructura, Densidad, Aireación, etc.). Fundamentos para la Interpretación de Análisis de Suelos, Plantas y Aguas para riego. Bogotá, Colombia: Sociedad Colombiana de la Ciencia del Suelo; 1995. p. 99.

17. Claver F. Guía para la elaboración de estudios del medio físico: contenido y metodología. Madrid: Centro de estudios de ordenación de territorio y medio ambiente, 1981.

18. SCCS. Sociedad Colombiana de la Ciencia del Suelo. Fundamentos para la interpretación de análisis de suelos, plantas y agua para riego. Bogota D.C2000.

19. Guerrero MR. La acidez del suelo, su naturaleza, sus implicaciones y su manejo. 1995. In: Fundamentos para la Interpretación de Análisis de Suelos, Plantas y Aguas para riego [Internet]. Bogota: Sociedad Colombiana de la Ciencia del Suelo; [141].

20. Pumisacho M, Sherwood S. El cultivo de la papa en Ecuador: Editorial Abya Yala; 2002. 